\title{
Medición de Oximetría de Pulso mediante Imagen fotopletismográfica.
}

\author{
Juan-Carlos Cobos-Torres \\ Universidad Carlos III de Madrid-Leganés, jcobos@ing.uc3m.es \\ Jordan Ortega Rodríguez \\ Universidad Carlos III de Madrid-Leganés, joortega@ing.uc3m.es \\ Pablo J. Alhama Blanco \\ Universidad Carlos III de Madrid-Leganés, palhama@pa.uc3m.es \\ Mohamed Abderrahim \\ Universidad Carlos III de Madrid-Leganés, mohamed@ing.uc3m.es
}

\begin{abstract}
Resumen
El presente método permite realizar la medición de la oximetría de pulso sin contacto por la técnica de imagen fotopletismográfica (FPGI). El filtro de Kalman se usa para reducir las frecuencias bajas de las señales FPGI. El método tiene características que lo convierten en una innovación en el campo. El paciente no tiene ningún contacto con el dispositivo de medición, y el algoritmo desarrollado tiene un requerimiento computacional bajo. Además, el método tiene una alta tolerancia a los artefactos. El enfoque analiza señales pletismográficas recogidas a través de una cámara de color estándar para estimar la oximetría de pulso. Los promedios de cada trama par e impar crean dos señales. Cada trama está sincronizada con una iluminación multiplexada de longitud de onda específica 640 y $950 \mathrm{~nm}$ respectivamente. El presente estudio ha obtenido una buena estimación de la oximetría de pulso para diez sujetos. El análisis de Bland-Altman muestra una fuerte correlación entre un sistema estándar y la medición FPGI.
\end{abstract}

Palabras Clave: Imagen Fotopletismográfica, Filtro de Kalman, Frecuencia Cardíaca, Saturación de oxígeno, Medición en línea.

\section{INTRODUCCIÓN}

El oxímetro de pulso consta de dos diodos emisores (el primero, emite luz roja a aproximadamente 650 nm; el segundo, emite luz infrarroja a aproximadamente $920 \mathrm{~nm}$ ) y un detector de fotodiodos [1], [2]. Una carcasa de plástico cubre los diodos, el fotodiodo y el miembro de la persona para evitar la interferencia de fuentes de luz distintas a la luz del dispositivo. Esta carcasa también permite la sujeción y el posicionamiento correcto del oxímetro. Debido a su facilidad de uso y colocación, la oximetría de pulso puede utilizarse fuera del contexto clínico (durante la anestesia [3], el cuidado crítico [4], el triaje [5], etc.). Por esta razón, son varios los métodos que han propuesto el uso de cámaras para medir la saturación de oxígeno $\left(\mathrm{SPO}_{2}\right)$. En primer lugar, los dispositivos que forman parte de nuestra vida cotidiana, tales como teléfonos móviles, tabletas y computadoras se pueden usar o emplear como dispositivos de medición. Además, estos dispositivos permiten la adquisición de vídeo en línea y cada trama puede procesarse, lo cual nos permite buscar y detectar cambios de color producidos por el flujo sanguíneo en los capilares de la piel.

Wieringa et al. [6], detallan la adquisición de latidos cardíacos a través de una cámara especial (frecuencia de muestreo de $100 \mathrm{~Hz}$ ) con un sensor CMOS monocromático y tres longitudes de onda diferentes (diodos LEDs que emiten luz a 660, 810 y $940 \mathrm{~nm}$ ). El estudio muestra la posible medición de la $\mathrm{SPO}_{2} \sin$ contacto a través de la reflexión de la luz. No se debe olvidar, una cámara monocromatica es mas sensible que una camara RGB por no contar con un filtro para cada color. Ademas, mediante la lente apocromática se concentran las tres longitudes de onda presentes en la ilumininacion utilizada en el foco del plano del sensor de la camara; por lo cual los latidos del corazón se encuentran presentes con las tres longitudes de onda.

Kong et al. [7], presentan otra metodología distinta que utiliza dos cámaras monocromáticas bajo iluminación regular para medir la $\mathrm{SPO}_{2}$ de la sangre (cada cámara tiene un filtro de banda estrecha de 660 $\mathrm{nm}$ y $520 \mathrm{~nm}$ ). El método utiliza dos longitudes de onda concretas que no siempre están presentes dentro del espectro de cada tipo de iluminación disponible, como ocurre por ejemplo en el caso de emplear LED blanco o luz fluorescente. Por lo tanto, el método sólo funcionará correctamente en entornos con luz natural. 
Otros métodos [8], [9] usan cámaras normales con sensores CMOS o CCD. Cada uno tiene diferentes configuraciones para la adquisición: respectivamente, frecuencia 12 y $20 \mathrm{fps}$, resolución 5 y 2 megapixeles, y distancia $100,40 \mathrm{~cm}$. Los métodos, calculan la $\mathrm{SPO}_{2}$ bajo una iluminación regular. Además, los muestran que la $\mathrm{SPO}_{2}$ se puede calcular a través de los canales rojo y azul de la cámara RGB; lo que cambia es la herramienta utilizada para la extracción de la señal fotopletismográfica. El primer método utiliza un proceso autorregresivo, es esencialmente un filtro de respuesta de impulso infinito (IIR). No es aconsejable diseñar directamente un filtro de paso bajo IIR que tenga una frecuencia de muestreo de banda de paso muy pequeña, ya que los polos deben estar agrupados muy cerca; entonces, un movimiento ligero de los polos dañará la respuesta de frecuencia deseada. El segundo método, (desarrollado por los mismos autores principales) utiliza el proceso ICA con un conjunto de modelos autorregresivos. Su principal inconveniente es un coste computacional elevado.

Sin embargo, los métodos anteriores sufren una limitación significativa que consiste en que sólo funcionan en entornos con iluminación natural o una iluminación excelente. Además, los métodos sólo funcionan en implementaciones fuera de línea porque sus algoritmos tienen un coste computacional elevado. Por otra parte, sólo el método presentado por Bal [10], utiliza un sistema automático para detectar la región de interés (ROI). La detección sufre un efecto de sacudida o agitación, ya que en la detección realizada por el metodo de Haar Cascade se observa este efecto claramente en el cuadro delimitador de la ROI. Este problema podría causar artefactos $o$ ruidos en la señal pletismográfica adquirida.

Por último, el método denominado Sofía [11], es interesante, ya que utiliza una metodología diferente. Utiliza promedios de múltiples ROIs; pero los autores, no explican el método de filtrado para las señales pletismográficas, ni especifican el uso de un filtro de eliminación de tendencia. Al parecer, se utiliza un filtro no causal de paso de banda que los autores explican en otro documento [12].

El presente método sin contacto propuesto, demuestra que es factible medir la $\mathrm{SPO}_{2}$ a través de una cámara de color estándar con un sistema elemental de iluminación. El sistema tiene su propia iluminación y por tanto funciona independientemente de la iluminación ambiental. Ademas, al utilizar un filtro kalman para el offset o la tendencia de la señal permite su uso para la medida de la frecuencia cardíaca y $\mathrm{SPO}_{2}$ de manera robusta y con un coste computacional bajo; características fundamentales que le permiten trabajar en línea.

\section{MATERIALES Y MÉTODOS}

Mediante una cámara a color estándar se captura la señal pletismográfica para estimar el pulso cardíaco y la $\mathrm{SPO}_{2}$ en la piel de un sujeto. El ROI seleccionado, es una pequeña región en la frente de una persona. Dos matrices de diodos emisores de luz se utilizan para iluminar el ROI (640 y $950 \mathrm{~nm})$; las cuales se denominaran Fuente de Luz Multiplexada (MLF). Los frames de la cámara se sincronizan con cada matriz de iluminación. Los componentes principales del sistema se representan en la Fig. 1. Por último, utilizamos un oxímetro de pulso médico para verificar la medición de la oximetría de pulso.

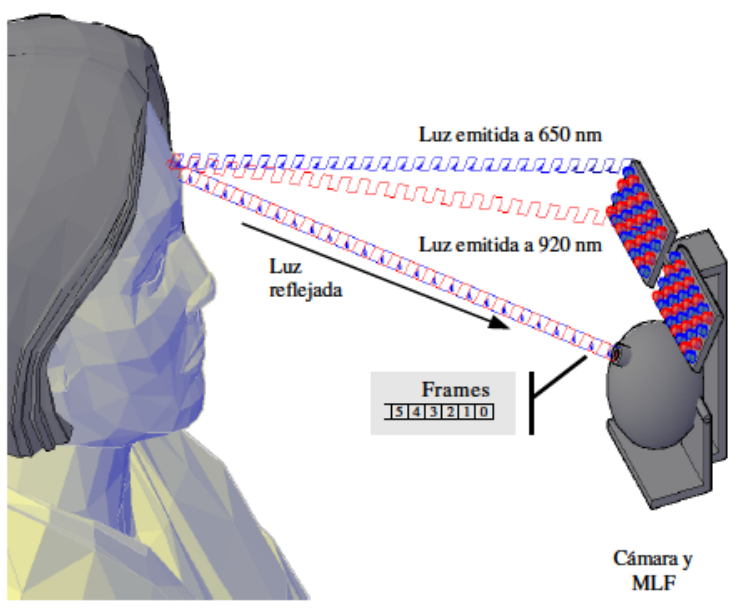

Figura 1: Configuración del experimento. Frames pares luz reflejada a $640 \mathrm{~nm}$ y frames impares luz reflejada a $950 \mathrm{~nm}$.

\subsection{HARDWARE}

La cámara utilizada es una cámara Web con sensor OV9710 HD CMOS, con un tamaño de sensor de 1/4'; proporciona una resolución WXGA (1280 x 800 ), con una frecuencia de captura máxima de 30 frames por segundo.

La MLF diseñada y construida utilizando 24 LEDS, formando una matriz intercalada. El primer grupo tiene 12 LEDs rojos con una longitud de onda pico de $640 \mathrm{~nm}$ aproximadamente y el segundo grupo tiene 12 LED IR con una longitud de onda pico de $950 \mathrm{~nm}$ aproximadamente (ambos tipos de LED tienen un tamaño T-1 3/4). La disposición de los LEDs rojo e IR se representa en la Fig. 1.

\subsection{POBLACIÓN}

La investigación se realizó de acuerdo con las regulaciones, principios y aprobaciones necesarias. En este experimento, participaron voluntariamente diez sujetos de edad y sexo diferente; para estructurar una muestra heterogénea, permitiendo demostrar la 
eficiencia del método propuesto. Durante las mediciones los sujetos estaban sentados frente a la cámara y a la MLF, ubicados a una distancia aproximadamente de $50 \mathrm{~cm}$.

\subsection{DISEÑO DEL EXPERIMENTO}

La luz reflejada detectada en la superficie de la piel ha viajado a través de las capas de la piel. El proceso se ilustra en la Fig. 2. Si se aplica una luz incidente a la piel, parte de esta luz es absorbida. La onda larga UV, el espectro visible y el infrarrojo cercano se dispersan hacia adelante a la dermis (la profundidad de absorción de la radiación está determinada por la longitud de onda); fibras de colágeno muy densas, agua y vasos sanguíneos componen la dermis [13]. Las fibras de colágeno dispersan la luz fuertemente. La capa de la epidermis produce retro-dispersión de una parte de la luz. La luz retro-dispersada ha pasado a través de los vasos sanguíneos, por lo que oxihemoglobina $(\mathrm{HbO} 2)$ y des-oxihemoglobina $(\mathrm{Hb})$ han absorbido algunas longitudes de onda de luz y la luz retro-dispersada ha sido modulada por el flujo sanguíneo pulsante, para seguir siendo dispersada hacia adelante al estrato córneo. La luz resultante se transmite y viaja a la cámara con la señal pletismográfica. Además, es importante recordar que la intensidad de la luz reflejada es inversamente proporcional al cuadrado de la distancia entre el MLF y la piel, así como entre la piel y la cámara.

La estructura general, utilizada para la obtención de la frecuencia cardíaca es detallada en la Fig. 2.

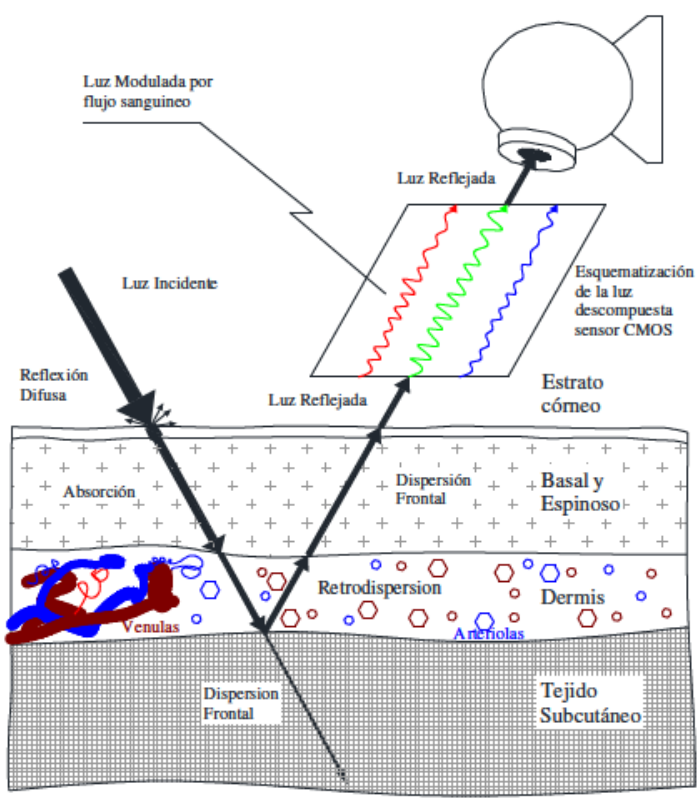

Figura 2: Interacción de la piel con una luz incidente.

La cámara captura la luz de la superficie de la piel y las imágenes se procesan en línea. El lenguaje de programación Python fue seleccionado para procesar las imágenes, junto con varias librerías. La librería OpenCV se utiliza para comunicarse con la cámara. Además, la librería Kazemi y Sullivan [14] permite la detección facial. Este detector utiliza un conjunto de árboles de regresión para la selección de la cara. Las características utilizadas son las cejas (principio y final). Con esta información, procedemos a estimar el área de análisis, corresponde a un área promedio de 1600 píxeles cuadrados (40 x 40 píxeles); cuando el sujeto se encuentra a una distancia estimada de $50 \mathrm{~cm}$ de la cámara. Dado que no todos los píxeles en el ROI seleccionado contienen la variación de brillo de la señal pletismográfica, los píxeles se combinan en un valor de brillo medio único. Los frames de la cámara se sincronizan con cada matriz de iluminación. Cada matriz ilumina diferentes frames capturados por la cámara (par e impar). Después de calcular el promedio de ROI, se generan las pilas tipo FIFO (primero en entrar, primero en salir). De este modo, se obtienen dos señales pletismográficas a través del mismo canal del sensor de la cámara (canal rojo); este es el principal requisito para estimar la $\mathrm{SPO}_{2}$.

\subsection{CALIBRACIÓN DE LA FUENTE DE LUZ MULTIPLEXADA}

Se prestó la debida atención a la seguridad de los ojos y la piel. La potencia radiada de los LEDs y/o IR-LEDs y/o el tiempo de exposición pueden afectar la piel o los ojos de diferentes maneras. Un tiempo largo de exposición o una alta radiación podrían causar una destrucción celular por sobrecalentamiento del tejido irradiado; dando como resultado un daño permanente al tejido. Bajo estos parámetros de seguridad se diseñó y construyó el MLF, bajo la norma IEC-62471.

El MLF se compone de la matriz de 12 x IR LEDs y $12 \mathrm{x}$ LEDs rojos. La primera matriz tiene una operación pulsada de impulso IF $=100 \mathrm{~mA}$ (corriente directa), longitud de onda pico $950 \mathrm{~nm}$. La segunda serie tiene una operación pulsada de IF $=10 \mathrm{~mA}$, longitud de onda pico $640 \mathrm{~nm}$. Ambos matrices tienen tiempo de pulso $\mathrm{tp}=16,5 \mathrm{~ms}$, ciclo de servicio $\mathrm{D}=0,5$ para fines de irradiación, distancia mínima para el usuario $r>0,5 \mathrm{~m}$ para iluminación de cara, tamaño de matriz de $0,3 \times 0,3 \mathrm{~mm}^{2}$, tiempo de exposición $\mathrm{t}>1000 \mathrm{~s}$.

\subsection{ALGORITMO DE PROCESAMIENTO}

El esquema general adoptado para el procesamiento de las imágenes, estimación de la frecuencia cardíaca y la $\mathrm{SPO}_{2}$ se ilustra en la Fig. 3. Las mediciones son adquiridas en línea. Como primer paso, la cámara y MLF se sincronizan. Un microcontrolador Arduino 
hace parpadear la matriz de LEDs y se comunica a través de una interfaz serie, usando el código Python.

A continuación, las marcas faciales se encuentran desde el primer frame. La posición de la cabeza (tono, guiñada y alabeo) se calcula con las marcas de las cejas (comienzo y final). El principio y el final de las cejas definen el ROI en forma de rectángulo. La forma ROI se selecciona en una posición paralela a la línea imaginaria que conecta las cejas (caso de alabeo). El tamaño de la ROI depende de la distancia entre las cejas (además, también depende de la distancia de la cara a la cámara). Asimismo, el rectángulo crece horizontalmente en la dirección opuesta a la del movimiento de la cara (guiñada) y en ambas direcciones (tono). Una vez que se define la ROI, sus píxeles se combinan en un sólo valor promedio. Con cada nuevo promedio de cada trama, generamos dos pilas FIFO. Los frames pares generan la primera pila (trama iluminada a $640 \mathrm{~nm}$ ) y los frames impares generan la segunda pila (trama iluminada a $950 \mathrm{~nm}$ ).

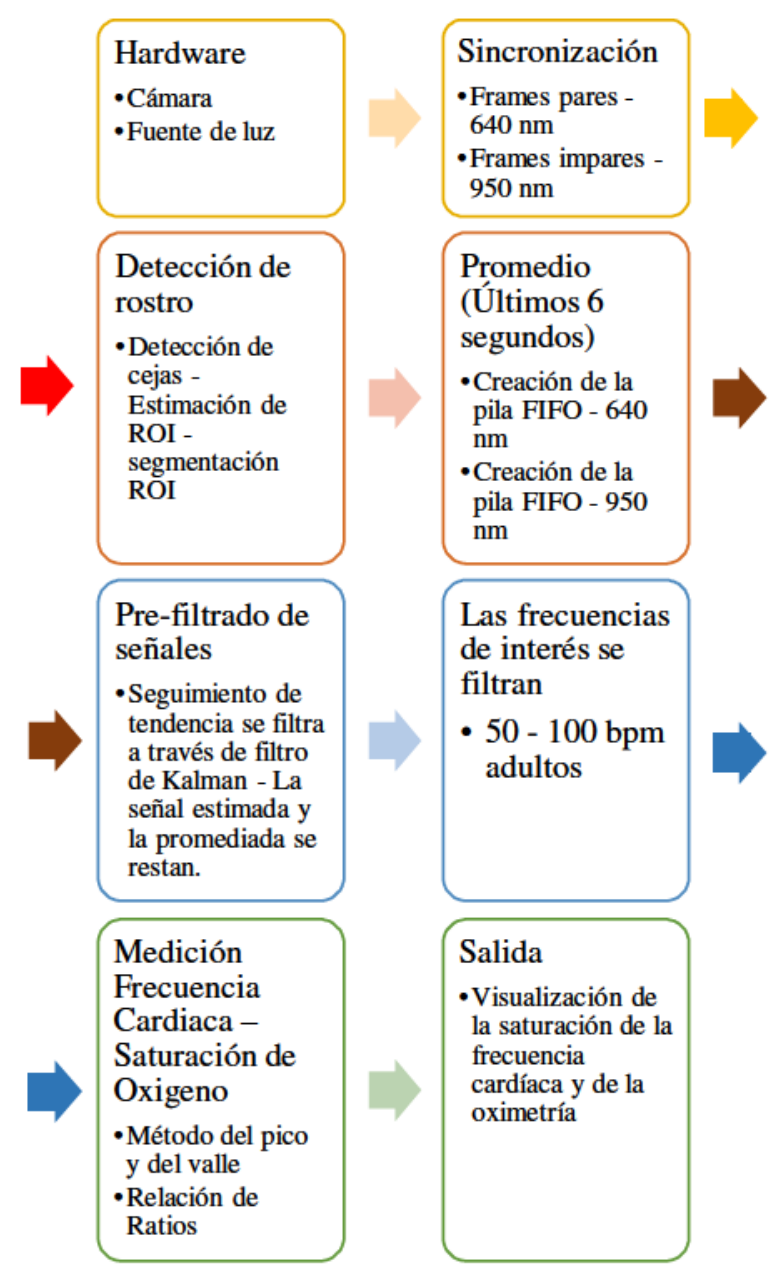

Figura 3: Secuencia utilizada para las mediciones

La dimensión de la pila es de 6 segundos. Este valor se selecciona porque es lo suficientemente largo como para tener un mínimo de seis pulsos cardíacos. Después de llenar las pilas, el método de Kalman estima el desplazamiento de las señales (seguimiento de tendencia). Se genera un filtro de frecuencia baja cuando se restan las señales promedio y de estimación. Este filtro es muy estable y sensible a los cambios en la señal. Además, tiene un tiempo de estabilización inicial muy rápido con un coste computacional bajo.

\section{RESULTADOS Y DISCUSIÓN}

Se compararon los valores de las medidas, obtenidas mediante un oxímetro de pulso de dedo convencional y el método de medición sin contacto propuesto. Los sujetos respiraban espontáneamente cuando los valores fueron medidos. El grado de acuerdo para las mediciones se calcula usando las mediciones tomadas de diez sujetos, como se muestra en la Fig. 4. La figura muestra que los resultados con el dispositivo sin contacto son comparables con los del dispositivo de contacto como monitor de pulsimetría Fig. 4a y $\mathrm{SPO}_{2}$. Fig. 4b.
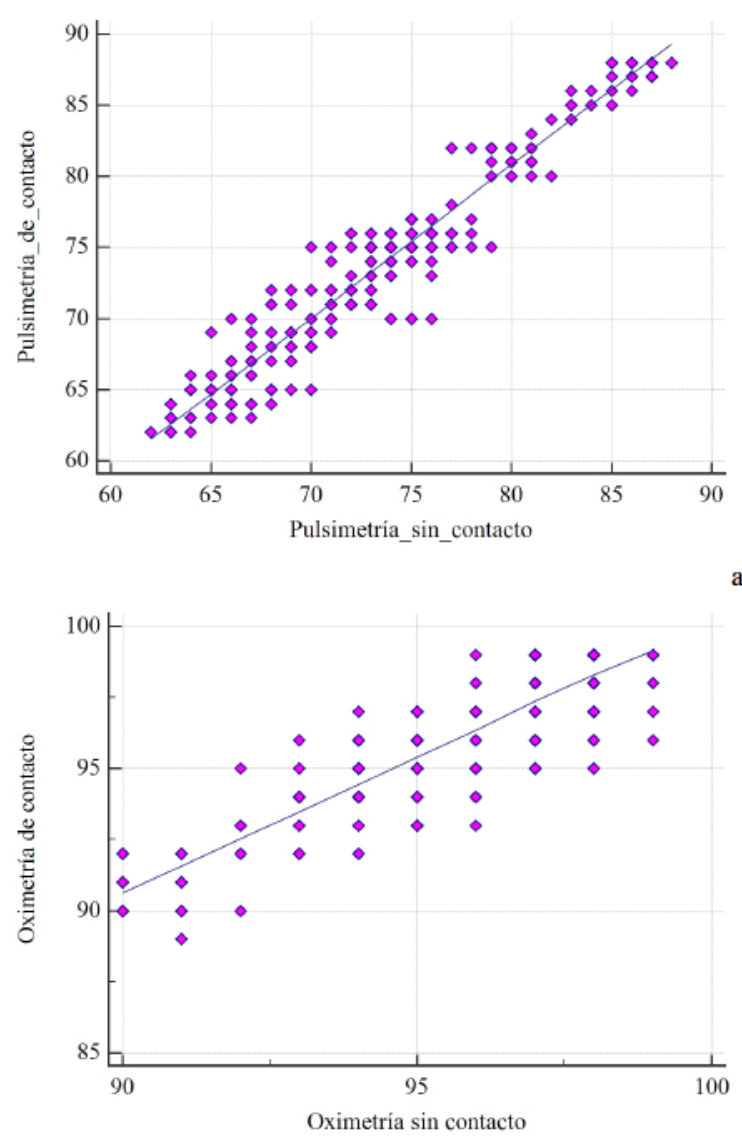

b)

Figura 4: Diagrama de dispersión que muestra la relación entre las medidas realizadas con el método de contacto y el método propuesto sin contacto (a) Pulso cardíaco, (b) $\mathrm{SPO}_{2}$. 
Además, se analizaron los resultados mediante el método de Bland-Altman [15] con el fin de confirmar el rendimiento y la fiabilidad del sistema. Las diferencias entre las medidas por contacto y sin contacto se representan en función de los promedios de ambos sistemas para la pulsimetría y la oximetría, Fig. 5. Los promedios están representados por líneas de puntos; $95 \%$ de los límites de acuerdo ( $\pm 1,96$ SD) se representan por líneas de trazos. Específicamente, el sesgo medio fue de 0,3 con límites de acuerdo del $95 \%,-3,3$ a 3,9 bpm para el pulso cardíaco. Y para la $\mathrm{SPO}_{2}$, el sesgo medio fue de 0,3 con límites de acuerdo del 95\%, -2,1 a 2,7\%. La desviación estándar de los residuos es de 1,83 para el pulso cardíaco y de 1,23 para el $\mathrm{SPO}_{2}$.
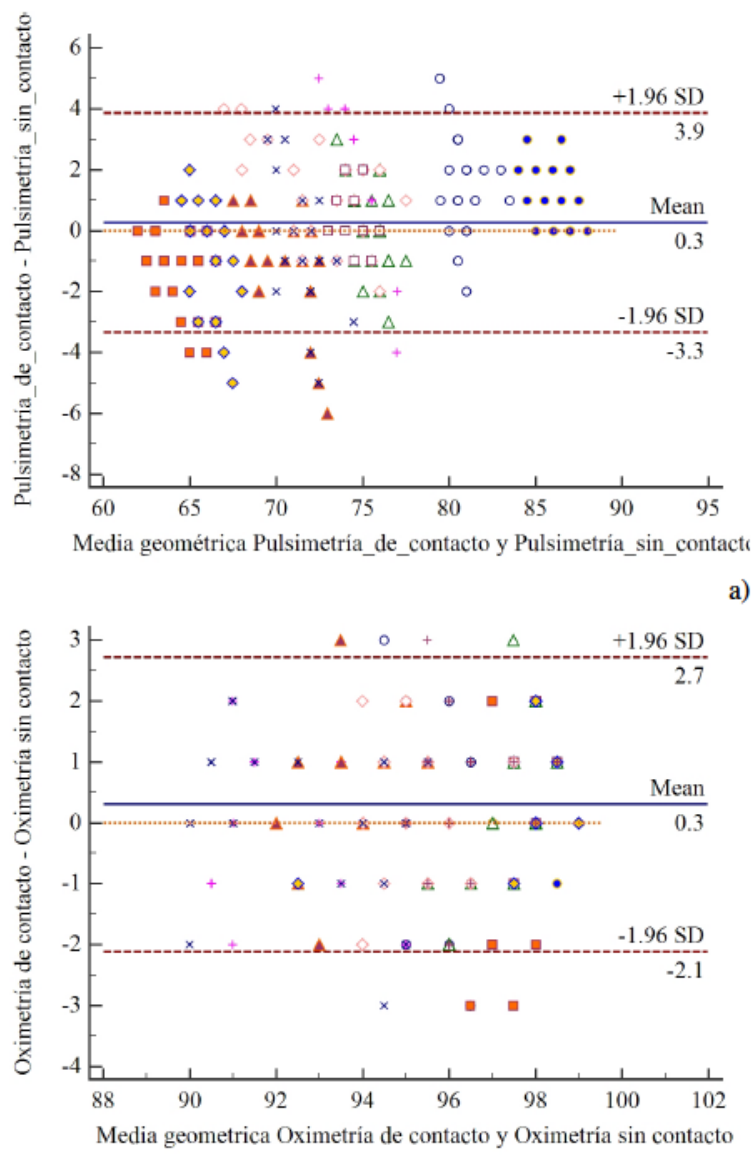

Figura 5: Gráficos de Bland-Altman que muestra el nivel de acuerdo entre las medidas realizadas con el método de contacto y el método propuesto sin contacto. (a) Pulso cardíaco, (b) $\mathrm{SPO}_{2}$.

La medida remota de la pulsimetría y oximetría a través de una cámara de color estándar y MLF fue investigada en este trabajo. Hemos diseñado, implementado y evaluado un método para obtener la frecuencia cardíaca así como la $\mathrm{SPO}_{2}$. El método funciona a partir de imágenes capturadas de la cara de un sujeto, adquiridas y procesadas en línea.
Finalmente, se evalúa el rendimiento del sistema; los resultados muestran un buen acuerdo entre las medidas realizadas por el método propuesto y las medidas obtenidas mediante un sensor de oxímetro de pulso comercial. El MLF utilizado, permite que el dispositivo esté libre de cualquier interferencia producida por la luz ambiente. Además, la mayoría de los métodos existentes tienen un costo computacional muy alto, y los pocos que están diseñados para trabajar en línea, también son muy susceptibles a artefactos (variación de iluminación y/o movimiento de personas). Todas estas deficiencias han sido ampliamente superadas en el método presentado aquí. Aunque, el alcance del presente trabajo se limita a la recuperación de la frecuencia cardíaca y la $\mathrm{SPO}_{2}$, muchos otros parámetros fisiológicos importantes pueden medirse usando las mismas imágenes.

\section{CONCLUSIONES}

El presente método permite medir la pulsimetría y la $\mathrm{SPO}_{2}$ sin contacto de un sujeto; el método es robusto a movimientos o artefactos. Además, los autores estiman que el método propuesto se caracteriza por un algoritmo de costo computacional muy bajo; permitirá su implementación para mediciones en línea de pulsimetría y oximetría en dispositivos móviles estándar.

Como ya se mencionó anteriormente, el presente sistema de medición de pulsimetría y oximetría puede convertirse en base para los sistemas que funcionen por imagen fotopletismográfica, por su coste computacional bajo, su gran robustez y eficacia.

Actualmente, se trabaja en la medición de otros parámetros fisiológicos. Se espera en el futuro, seguir mejorando las mediciones mediante el análisis en paralelo de diversas regiones de la piel, un sistema de ponderación y comparación de dichas señales. Finalmente, se está evaluando al método propuesto frente a otros métodos por PPGI y en situaciones más reales, como la medición de estos dos signos vitales de un sujeto en movimiento junto con el sistema y a la vez cambiando de ambientes e iluminación.

\section{Agradecimientos}

El trabajo ha sido financiado parcialmente por la Acción Estratégica en Robótica, Visión por ordenador y Automatización; por el proyecto RoboCity2030-III-CM (Robótica aplicada a la mejora de la calidad de vida de los ciudadanos. fase III; S2013/MIT-2748) financiado por Programas de Actividades I+D en la Comunidad de Madrid y cofinanciado por los Fondos Estructurales de la Unión Europea. 
El primer autor agradece al Gobierno Ecuatoriano, por la beca que apoya y financia sus estudios. Su gratitud también a la ayuda directa de los laboratorios de la Universidad Carlos III de Madrid.

\section{Referencias}

[1] W. G. Zijlstra, A. Buursma and W. P. Meeuwsen-van der Roest. "Absorption spectra of human fetal and adult oxyhemoglobin, de-oxyhemoglobin, carboxyhemoglobin, and methemoglobin". Clinical. Chemestry. Vol. 37, No, 9, pp. 1633-1638, Sep. 1991.

[2] Y. Mendelson. "Pulse oximetry: Theory and applications for noninvasive monitoring", Clinical Chemestry, Vol. 38, No. 9, pp. 1601-1607, Sep. 1992.

[3] S. Sugino, N. Kanaya, M. Mizuuchi, M. Nakayama and A. Namiki. "Forehead is as sensitive as finger pulse oximetry during general anesthesia", Canadian Journal of Anesthesia, Vol. 51, No. 5, pp. 432-436, May 2004.

[4] A. Jubran. "Pulse oximetry", Critical Care, Vol. 3, No. 2, pp. R11, May 1999.

[5] W. R. Mower, C. Sachs, E. L. Nicklin, P. Safa and L. J. Baraff. "Effect of routine emergency department triage pulse oximetry screening on medical management", Chest, Vol. 108 , No, 5, pp. 1297-1302, Nov. 1995.

[6] F. P. Wieringa, F. Mastik and van der Steen, Antonius FW. "Contactless multiple wavelength photoplethysmographic imaging: A first step toward -SpO2 camera- technology", Annals of biomedical engineering, Vol. 33, No. 8, pp. 10341041, Mar. 2005

[7] L. Kong, Y. Zhao, L. Dong, Y. Jian, X. Jin, B. Li, Y. Feng, M. Liu, X. Liu and H. Wu. "Non-contact detection of oxygen saturation based on visible light imaging device using ambient light”, Optics Express, Vol. 21, No. 15, pp. 17464 17471, 2013.

[8] L. Tarassenko, M. Villarroel, A. Guazzi, J. Jorge, D. Clifton and C. Pugh. "Non-contact video-based vital sign monitoring using ambient light and auto-regressive models", Physiological measurement, Vol. 35, No. 5, pp. 807, Mar. 2014.

[9] M. Villarroel, A. Guazzi, J. Jorge, S. Davis, P. Watkinson, G. Green, A. Shenvi, K. McCormick and L. Tarassenko. "Continuous non-contact vital sign monitoring in neonatal intensive care unit", Healthcare Technology Letters, Vol. 1, No. 3, pp. 87-91, Sep. 2014.

[10] U. Bal. "Non-contact estimation of heart rate and oxygen saturation using ambient light", Biomedical Optics Express. Vol. 6, No. 1, pp. 86-97, 2015.

[11] A. R. Guazzi, M. Villarroel, J. Jorge, J. Daly, M. C. Frise, P. A. Robbins and L. Tarassenko. "Non-contact measurement of oxygen saturation with an RGB camera", Biomedical Optics Express, Vol. 6, No. 9, pp. 3320-3338, 2015.

[12] M. Kumar, A. Veeraraghavan and A. Sabharwal. "DistancePPG: Robust non-contact vital signs monitoring using a camera", Biomedical Optics Express, Vol. 6, No. 5, pp. 1565-1588, 2015.

[13] R. Henderson and K. Schulmeister. "Laser Safety", Institute of Physics Publishing, London, 2004.

[14] V. Kazemi and J. Sullivan. "One millisecond face alignment with an ensemble of regression trees", Presented at Proceedings of the IEEE Conference on Computer Vision and Pattern Recognition, 2014.

[15] J. M. Bland and D. Altman. "Statistical methods for assessing agreement between two methods of clinical measurement”, The Lancet, Vol. 327, No. 8476, pp. 307-310, 1986. 